\title{
Study on the Identification of Influencing Factors of Interactive Development between Innovation and Urbanization
}

\author{
Bo YUAN ${ }^{1, a}$, Feng-Chao LIU ${ }^{1, b, *}$ \\ ${ }^{1}$ Faculty of Management and Economics, Dalian University of Technology, China \\ a100607062@qq.com, bfengchaoliu@126.com \\ ${ }^{*}$ Corresponding author
}

Keywords: Innovation, Urbanization, Interactive Development, Influencing Factors

\begin{abstract}
By improving the disadvantages of traditional DEMATEL method, this study proposes an identification model of influencing factors based on Entropy - DEMATEL algorithm. Then, take China as research example, this study selects 20 factors related to the interactive development between innovation and urbanization, and empirically examines the influencing factors of interactive development between innovation and urbanization using the Entropy-DEMATEL algorithm. By analyzing the influencing degree, influenced degree, central degree and causal degree of each factor, we reveal the role of each factor in the interactive development process.
\end{abstract}

\section{Introduction}

It has been clearly demonstrated in the 18th CPC National Congress that technology innovation is a strategic support to improve social productivity and national comprehensive strength, which must be put in the core position of overall national development. The Congress also emphasizes that we should stick to the road of independent innovation with Chinese characteristics and the implementation of innovation-driven strategy. Meanwhile, the new type of urbanization proves to be a major strategy put forward in the 18th National Congress and a necessary way to achieve economic development and technological progress. It has been indicated by many existing studies that, while science and technology innovation is a strong power to propel the development of a city, urbanization accelerates the emergence and development of urban innovation and is assumed to be a key factor to promote technical creations. The development of urban and the innovation of technology always have a mutual promotion and rely on each other all the time. Technology innovation is a great power of human progress while urbanization facilitates the generation of innovation, so both of them can influence each other. Analyzing the interactive development of innovation and urbanization helps to identify the developing status and the influencing degree of them, which could provides theoretical guidance for the innovation-driven development and urbanization. In order to analyze the interactive development of technology innovation and urbanization, we should identify the influencing factors of the interactive development firstly, and then examine the complex relations between them based on the influencing factors. Therefore, this study combined entropy method and DEMATEL algorithm to identify the influencing factors of interactive development between innovation and urbanization, and discuss the influencing direction and the similarities and differences of those factors.

\section{The Identification Method of Influencing Factors}

\section{DEMATEL Algorithm}

The DEMATEL algorithm, regarded as Decision-Making and Trail Evaluation Laboratory algorithm, is an effective research technique to identify and analyze the mutual influence mechanism of system factors by using graph theory and matrix. It is a methodology proposed by the United States Bastille National Laboratory in 1971, which applied graph theory and matrix theory to analyze system factors. The specific steps are as follows: 
(1) Identify the influencing factors: According to the practical research problem, we identify the factors that influence a system by using the system diagnostic methodology.

(2) Establish the relationship of factors: First of all, we refer to some relative literatures; next, we draw a directed graph according to the relationship and mark the influencing degree numerically.

(3) Construct a direct impact matrix according to the relationship between each two factors: Generally we choose capital letters to represent for the direct impact matrix, for example, $Y=\left[Y_{i j}\right]_{N, N}, \mathrm{~N}$ refers to the number of factors, while $Y_{i j}$ means the direct influencing degree that factor i imposes on factor $\mathrm{j}$,

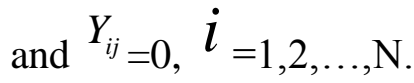

(4) Standardize the direct impact matrix: In order to better analyze the indirect influencing relationship among factors, we need to standardize the direct impact matrix. The processes are as follows: Firstly, we calculate the sum of each row and find the maximum value; secondly, we divide the direct impact matrix by the maximum and we can get an indirect impact matrix X.

(5) Establish the comprehensive impact matrix: Calculate the eigenvalue of $X$, and under the condition that the maximum of eigenvalue is less than 1 , calculate the comprehensive impact matrix $\mathrm{T}$, $T=X(I-X)^{-1}$

(6) According to the comprehensive impact matrix $\mathrm{T}$ and the elements $T_{\mathrm{ij}}$, we can identify the influencing relationships among the factors and come to a conclusion about the influencing degree and influenced degree of each factor, which could be used to calculate the central degree and causal degree.

Here, the value of $T_{\mathrm{ij}}$ represents the direct and indirect influencing degree which factor i brings to j, or the degree that factor $\mathrm{j}$ been influenced comprehensively by $\mathrm{i}$. The sum of each row in matrix $\mathrm{T}$ means the comprehensive influence value that the elements of every row acts on all other elements, which is also regarded as influencing degree, demonstrating the ability to influence others. Meanwhile, the sum of each column in matrix T means the comprehensive influence value that the elements of every column acts on all other elements, which is also regarded as influenced degree, demonstrating the degree been influenced by other factors. When the causal degree is greater than 0 , which means the element has a greater impact on others, it is known as influencing factor; otherwise, when the causal degree is less than 0 , which means the element has been greatly influenced by others, we define it as influenced factor.

\section{Entropy-DEMATEL Algorithm}

Traditional DEMATEL algorithm is too subjective when experts are scoring; however, it is not always the case in reality. In order to solve this problem and construct a more objective decision-making model, this study trying to use entropy method to figure out the direct correlation matrix among indices. On the one hand, this research takes the differences of different provinces during the process of interactive development between innovation and urbanization into consideration as variables, making the model more reasonable. On the other hand, it also solves the problem of subjectivity in expert assignment, which makes the evaluation results more close to the actual value. This study combines entropy method and DEMATEL algorithm to analyze the influencing factors of interactive development between innovation and urbanization. The process is as follows:

(1) Calculate the index weight ${ }^{w_{j}}$ of innovation and urbanization by using entropy algorithm, while $w_{j}$ represents for the index weight that factor $\mathrm{j}$ imposed on the interactive development between innovation and urbanization.

(2) Calculate the direct correlation matrix of influencing factors: 


$$
\begin{aligned}
& B=\left(b_{i j}\right)_{n \times n}=\left\{\left(\begin{array}{ccc}
b_{11} & \cdots & b_{1 n} \\
\vdots & \ddots & \vdots \\
b_{n 1} & \cdots & b_{n n}
\end{array}\right)\right\} \\
& \text { Therein, } b_{i i}=0, b_{i j}=\frac{w_{i}}{w_{j}} \text { refers to the importance that factor i exerts on } \mathrm{j} .
\end{aligned}
$$

(3) Normalize the direct correlation matrix:

$$
X=\left(x_{i j}\right)_{n \times n}=\frac{1}{\max _{1 \leq i \leq n} \sum_{j=1}^{n} b_{i j}} \bullet B
$$

(4) Calculate the comprehensive correlation matrix:

$$
T=X(I-X)^{-1}
$$

Therein, $(I-X)^{-1}$ is the inverse matrix of $I-X, I$ represents for unit matrix.

(5) Establish a causality diagram. Define D as the sum of each row of matrix T, while define $\mathrm{R}$ as the sum of each column of matrix T:

$$
\begin{aligned}
& T=\left(t_{i j}\right)_{n \times n} \\
& D=\left(t_{i}\right)_{n \times 1}=\left(\sum_{j=1}^{n} t_{i j}\right) n \times 1 \\
& R=\left(t_{j}\right)_{1 \times n}=\left(\sum_{i=1}^{n} t_{i j}\right) 1 \times n
\end{aligned}
$$

We define $D_{i}+R_{i}$ as central degree of index $\mathrm{i}$, and the larger the number is, the more important it will be. We also define $D_{i}-R_{i}$ as causal degree to distinguish the reasons group and results group: index $\mathrm{i}$ is considered in reasons group when $D_{i}-R_{i}$ is weighed larger than 0 ; otherwise, it is considered in results group. Among numerous influencing factors, factors in results group are the influenced results of factors in reasons group.

The advantage of this algorithm is that we replace the questionnaire and experts scoring in traditional DEMATEL algorithm by applying entropy method to calculate index weight when we get the correlation matrix among every factor. Therefore, it is able to avoid the subjective influence on evaluation results and make the analysis more credible by applying entropy to solve matrix.

\section{Indicator Selection and Data Sources}

Based on the characteristics of interactive development between innovation and urbanization and related literatures, we identify 20 indicators as the influencing factors, such as per capita GDP , R\&D investment intensity, per capita level of education, Internet users per million, per capita of local fiscal revenue and gross output value of industries above scale, per capita social total retail of consumables, full-time equivalent of $R \& D$ employees, technical personnel in enterprises and $R \& D$ personnel in research and development institutions, proportions of secondary and tertiary industries in GDP, proportions of secondary and tertiary industries employees and the non-agricultural population density, the actual use of foreign capital and the total amount of import and export, per capita investment in fixed assets, the number of patent authorization and high school subjects.

The data of this study comes from China Statistical Yearbook on Science and Technology, China City Statistical Yearbook, China statistical yearbook and the national statistical bulletin, which guarantees the objectivity and authority of the data used. 


\section{Empirical Results Analysis}

First of all, we calculate the index weight of each factor by using entropy method. Then applying DEMATEL algorithm to construct a direct correlation matrix and calculate the final influencing degree, influenced degree, central degree and causal degree of the influencing factors, the results are shown in Table1.

(1) Analysis based on causal degree

Causal degree reflects the degree of driving factors exerts on others. The larger the value is, the greater the degree of influence it will impose on others. What is considered as influencing factors of interactive development between innovation and urbanization is that, when the influencing degree of an indicator is larger than the influenced degreed, and it is less susceptible to change according to the outside environment.

Among all the influencing factors, the most important factor in causal degree is the number of high school subjects, followed by the total amount of import and export, however, the least important factor is the technical personnel in enterprises. Increasing investment in science and technology is determined by the level of productivity ultimately, while the productivity improvement also depends on the progress of science and technology. Therefore, increasing investment in science and technology and improving agricultural productivity can provide not only a lot of agricultural byproduct for urbanization development but also some necessary labor resources for non-agricultures at the same time. Non-agriculture industries, especially industrial science and technology development, could stimulate the formation and development of urban economic center; as a result, we can change the mode of economic growth from extensive to intensive fundamentally, and improve the social productivity level of a town.

What is considered as influenced factors of interactive development between innovation and urbanization is that, when the influencing degree of an indicator is less than the influenced degreed, and it is a media when influencing factors function. Meanwhile, influenced factors are always vulnerable to outside environment, so it is the most obvious influencing factors in the short term.

Among the influenced factors, the most important factor in causal degree is the proportion of tertiary industry personnel, and the least important factor is the per capita of education level. If we take industry as the first driving force of urbanization, the tertiary industry is the second one in the same process since one of its trait is labor-intensive, which possesses a great potential in absorbing labor resources. With the development of agricultural modernization and the opening of labor market, there may be a great deal of surplus labor force released from agriculture. In non-agriculture industries, whether city industries or rural industries, there always exists a problem which is about the limited capacity in absorbing labor resources. But with the further reform in state-owned enterprises, there will be a great many laid-offs in our society, which could exert a lot of pressure on labor market. Therefore, the third industry needs to have a breakthrough in the future.

(2) Analysis based on central degree

The larger the central degree value, the stronger effect it has imposed on the innovation and urbanization interactive development, which remains the most significant reasons when pushing the process. Otherwise, we regard that the elements remain in a secondary position and never even considered. The most important factor in this proceeding is the proportion of tertiary industry personnel, followed by the proportion of secondary and tertiary industries in GDP, while the least is the technical employees in enterprises. Whether to optimize the industrial structure or not will directly affect the level of urbanization since it is the core part and basis of urban economic structure.

At the same time, a reasonable industrial structure may be able to absorb labor sources to transfer from agriculture to non-agricultural industries effectively, thus to improve the urbanization level efficiently. 
Table 1 Analysis Results of influencing factors of interactive development between innovation and urbanization

\begin{tabular}{|c|c|c|c|c|c|}
\hline Num & Influencing Factors & $\begin{array}{l}\text { Influencing } \\
\text { degree(D) }\end{array}$ & $\begin{array}{l}\text { Influenced } \\
\text { degree(R) }\end{array}$ & $\begin{array}{c}\text { Central } \\
\text { degree }(\mathrm{M})\end{array}$ & $\begin{array}{c}\text { Causal } \\
\text { degree }(\mathrm{U})\end{array}$ \\
\hline 1 & GDP per capita & 0.1730 & 0.0534 & 0.2264 & $0.1196(9)$ \\
\hline 2 & $R \& D$ investment intensity & 0.1498 & 0.0618 & 0.2116 & $0.0879(12)$ \\
\hline 3 & Per capita level of education & 0.0802 & 0.1160 & 0.1962 & $-0.0359(7)$ \\
\hline 4 & Internet users per million & 0.1674 & 0.0552 & 0.2226 & $0.1122(10)$ \\
\hline 5 & Per capita of local fiscal revenue & 0.2789 & 0.0324 & 0.3113 & $0.2465(7)$ \\
\hline 6 & $\begin{array}{l}\text { Per capita social total retail of } \\
\text { consumables }\end{array}$ & 0.1534 & 0.0604 & 0.2138 & $0.0931(11)$ \\
\hline 7 & Non-agricultural population density & 0.0215 & 0.4079 & 0.4294 & $-0.3864(4)$ \\
\hline 8 & $\begin{array}{l}\text { Full-time equivalent of R\&D } \\
\text { employees }\end{array}$ & 0.3417 & 0.0261 & 0.3678 & $0.3156(5)$ \\
\hline 9 & Technical personnel in enterprises & 0.1061 & 0.0877 & 0.1938 & $0.0184(13)$ \\
\hline 10 & Total amount of import and export & 0.6995 & 0.0116 & 0.7111 & $0.6878(1)$ \\
\hline 11 & $\begin{array}{l}\text { Proportion of secondary industries } \\
\text { in GDP }\end{array}$ & 0.0089 & 0.8754 & 0.8842 & $-0.8665(2)$ \\
\hline 12 & $\begin{array}{l}\text { Proportion of secondary industries } \\
\text { employees }\end{array}$ & 0.0250 & 0.3555 & 0.3805 & $-0.3305(5)$ \\
\hline 13 & $\begin{array}{l}\text { Gross output value of industries } \\
\text { above scale }\end{array}$ & 0.3236 & 0.0277 & 0.3513 & $0.2960(6)$ \\
\hline 14 & $\begin{array}{l}\text { Proportion of tertiary industries } \\
\text { employees }\end{array}$ & 0.0071 & 1.0404 & 1.0475 & $-1.0333(1)$ \\
\hline 15 & The actual use of foreign capital & 0.3536 & 0.0251 & 0.3787 & $0.3284(4)$ \\
\hline 16 & Per capita investment in fixed assets & 0.0585 & 0.1582 & 0.2167 & $-0.0997(6)$ \\
\hline 17 & The number of patent authorization & 0.5481 & 0.0155 & 0.5635 & $0.5326(2)$ \\
\hline 18 & $R \& D$ personnel in $R \& D$ institutions & 0.4167 & 0.0210 & 0.4377 & $0.3957(3)$ \\
\hline 19 & The number of high school subjects & 0.1925 & 0.0478 & 0.2403 & $0.1447(8)$ \\
\hline 20 & $\begin{array}{l}\text { Proportion of tertiary industry in } \\
\text { GDP }\end{array}$ & 0.0129 & 0.6393 & 0.6522 & $-0.6263(3)$ \\
\hline
\end{tabular}

\section{Conclusion}

This study examines the influencing factors of interactive development between innovation and urbanization based on the Entropy - DEMATEL algorithm, and comes to the following conclusions.

From the perspective of causal degree, the number of high school subjects shows to be the most fundamental driving factors of interactive development between innovation and urbanization, and finally turns out to be the sources of all the other factors. In a word, the number of high school subjects is a basic factor in innovation and urbanization interactive development.

From the perspective of central degree, the proportion of tertiary industry personnel is the most important factors influencing the interactive development between innovation and urbanization. It 
proves that the industrial structure is most crucial for improving the level of urbanization and technology innovation in China as well.

\section{Acknowledgement}

This research was financially supported by the National Natural Science Foundation of China (Grant No. 71173026, 71473026).

\section{References}

[1] S. Gu, J. Liu, Urbanization transfers from "factor driven" to "innovation driven", Population Research. 36, 6(2013) 3-12.

[2] S. Meng, The development path of new urbanization in China under innovation-driven strategy, Theoretical study. 7(2013) 27-29.

[3] R. P. Wilson, S. P. Vandenabeele, Technological innovation in archival tags used in seabird research, Marine Ecology Progress Series. 451, 4(2012) 245-262.

[4] A. Kijek, T. Kijek, The analysis of innovation input-output relationships in EU member states, Comparative Economic Research. 13, 3(2010) 93-106.

[5] M. Song, S. Im, H. Bij, et al. Does Strategic Planning Enhance or Impede Innovation and Firm Performance?, Journal of Product Innovation Management. 28, 4(2011) 503-520.

[6] P. Gao, X. Lu, Research on the expansion of the DEMATEL method and application based on interval number, Operations research and management. 23, 1(2014) 44-50.

[7] X. Xiang, P. Chen, A new grey relation measuring model based on entropy method, Journal of Systems Science. 22, 2(2014) 66-70. 